\title{
Sexual dysfunction related with carbamazepine treatment: how much do we ask?
}

\author{
Sibel Ustun Ozek ${ }^{1}$, Hazal Ceren $\mathrm{Ak}^{1 \oplus}$, Serap Ucler ${ }^{1 \odot}$ \\ ${ }^{1}$ Health Sciences University, Prof. Dr. Cemil Tascioglu City Hospital, Department of Neurology, Istanbul - Turkey
}

\section{Dear Editor,}

Hot water epilepsy is one of the reflex epilepsies (1). Most cases were reported from India and Turkey $(2,3)$. Pathogenesis is not clear, but regional prevalence may suggest genetic etiology. There are also case reports with a genetic locus (4). A possible mechanism for epileptogenesis may be a genetically abnormal thermoregulatory system or an anatomical abnormality of the temporo-insular and parietal networks (2). Seizures can be of focal or generalized onset. Seizures can be prevented to a great extent by changing bathing habits. Spontaneous remissions may also occur. Medical treatment may be required if seizure control cannot be achieved, or if non-reflex seizures occur apart from bathing. Among the medications used in the treatment are particularly carbamazepine, and sodium valproatevalproic acid, phenytoin, and phenobarbital (5). Antiepileptic treatments have frequent or rare side effects in the acute or chronic stage. There may also be side effects, such as sexual dysfunction, which can sometimes be overlooked if not questioned and can be difficult for the patient to express. Both epilepsy and the medications used play a role in the sexual dysfunction of the patients (6). Antiepileptic drugs can cause changes in hormones and cause sexual dysfunction. Phenobarbital, carbamazepine, and phenytoin can lead to direct suppression of gonadal testosterone synthesis and disruption of other peripheral sex steroid hormones by inducing liver enzymes (6). It has been reported that lamotrigine and levetiracetam, on the other hand, can significantly improve libido and orgasm (7). To reveal the prevalence of sexual function and when evaluating the patient's unresponsiveness to treatment, sexual functions should also be included in the side effect questionnaire form in cases of drug delay.

Thirty-nine-year-old, married for 19 years, primary school graduate male patient was admitted to the neurology outpatient clinic where he had been followed up with a diagnosis of hot water epilepsy. The history of the disease was described as a nonmotor seizure since the age of 8-9 years, in which awareness was impaired when hot water was poured directly on his head. He had a generalized seizure while bathing 9 years ago and two generalized seizures 3 months ago on the same day without any hot water stimulation. His neurological examination was normal. There was no additional disease in his history. In his family history, it was learned that his brother and aunt had similar stories. His brain MRI and sleep-wake EEG were normal. It was learned that he had continued carbamazepine treatment initiated in his seizure nine years ago for one month and that he ceased the treatment voluntarily considering he did not need treatment because of his rare seizures. During this period, he did not mention any side effects related to the drug. In his first outpatient application, carbamazepine $400 \mathrm{mg} /$ day was added to the treatment since he had non-reflexive and repetitive seizures during the day except bathing. At the follow-up examination three months later, the patient stated that he did not have seizures with treatment, used his medication regularly, but his only complaint was impotence, and

How to cite this article: Ustun Ozek S, Ak HC, Ucler S. Sexual dysfunction related with carbamazepine treatment: how much do we ask? Dusunen Adam The Journal of Psychiatry and Neurological Sciences 2020;33:328-330.

Correspondence: Sibel Ustun Ozek, Health Sciences University, Prof. Dr. Cemil Tascioglu City Hospital, Department of Neurology, Istanbul - Turkey E-mail: sibelustun@hotmail.com 
therefore he requested a change in treatment. His urological examination and psychiatric evaluation were normal. The total score he got from the Arizona Sexual Experiences Scale (ASEX) was 15. Considering the possible side effects of carbamazepine, the treatment was discontinued and it was changed to $600 \mathrm{mg} /$ day oxcarbazepine. The patient is still followed up without seizures and does not identify sexual dysfunction. In the control examination, his ASEX score decreased to 12. Written informed consent was obtained from the patient for the report.

Sexual dysfunctions are more common in patients with epilepsy than in the general population. While decreased libido is most common in women, hyposexuality and erectile dysfunction have been reported in men. In this group of patients, sexual dysfunction may occur due to many reasons. Structural abnormalities in the brain, hormonal causes, antiepileptic drugs, and psychosocial causes in patients with epilepsy may play a role in the etiology (8). Generally, patients with uncontrolled epilepsy, longer epilepsy duration, focal epilepsy, higher seizure frequency, and enzyme-causing and multiple antiepileptic drugs are more likely to have sexual dysfunction (9). Our case was married for 19 years and previously had no complaints regarding sexual functions. He was healthy urologically and psychiatrically. He had been receiving monotherapy regularly for 3 months and his seizures were under control.

Carbamazepine-related side effects can be considered as those occurring in the acute toxic state and those occurring in the subacute chronic period. Acute side effects are dizziness, blurred vision, diplopia, ataxia and balance disorder, which occur especially in toxic cases. Fever, lymphadenopathy and skin rash, leukopenia, anemia, thrombocytopenia, hyponatremia, and hepatotoxicity can be observed during the second week of the treatment (10). Enzyme-inducing drugs used in the treatment of epilepsy reduce the level of active testosterone by stimulating the aromatase and hepatic cytochrome $\mathrm{p}$ 450 enzymes. In studies conducted with carbamazepine reported a negative effect on hormonal levels and semen quality, and emphasized that it causes erectile dysfunction and a decrease in coital frequency (11). However, although the side effects related to carbamazepine are known, there is limited knowledge of the rate to which this returns as feedback from patients. With carbamazepine, a classic antiepileptic, this side effect is relatively less reported in daily practice. This includes the lack of questioning of healthcare professionals on this issue, time limitation, outpatient clinic conditions, as well as patient-induced embarrassment, and inability to express their complaints clearly. In our case, the patient clearly expressed his complaint and even stated it as the only complaint he had. Although the most common sideeffects are questioned in the control examination of epilepsy cases, those related to sexual function are neglected and questioning is not considered. However, the cases should be evaluated in all aspects and should be questioned thoroughly in a satisfactory time.

Oxcarbazepine, which is produced by the structural change of carbamazepine and has less metabolism with cytochrome $\mathrm{p} 450$, has not been defined, and there are reports that it is useful (12). In a study of 673 patients who received oxcarbazepine monotherapy, it was reported that there was an improvement in $79.4 \%$ of the cases and no change in $10.1 \%$ of the cases. This curative effect was found to be most noticeable in patients using enzyme-inducing drugs and whose treatment was re-arranged (13). In a meta-analysis investigating the effects of antiepileptic drugs on sexual functions, the healing effects of oxcarbazepine and lamotrigine were mentioned (8). Topiramate, pregabalin and gabapentin from the next generation of drugs cause sexual dysfunction, while oxcarbazepine, lamotrigine and levetiracetam have a healing effect (14). In our case, an improvement was identified when it was switched to oxcarbazepine. Hot water epilepsy is relatively rare. Although we interpret the side effect expressed by the patient as an incidental association, we believe that studies involving a large number of cases may be planned in this group of patients for pathogenesis and etiological clarification.

As a result, it is recommended to replace the etiology of sexual dysfunction if it is thought to be medicationrelated (14). Sexual dysfunctions may be one of the reasons that cannot be expressed in patients' treatment noncompliance. While assessing the cases, sexual function questionnaires should also be taken into consideration and patients should be questioned in detail and under appropriate conditions. Further prospective studies are needed to understand and determine the prevalence and treatment of antiepilepticassociated sexual dysfunctions.

Informed Consent: Written informed consent obtained.

Conflict of Interest: The author declares that there is no conflict of interest.

Financial Disclosure: No funding declared. 


\section{REFERENCES}

1. Blume WT, Lüders HO, Mizrahi E, Tassinari C, van Emde Boas W, Engel J Jr. Glossary of descriptive terminology for ictal semiology: report of the ILAE task force on classification and terminology. Epilepsia 2001; 42:1212-1218.

2. Satishchandra P. Hot-water epilepsy. Epilepsia 2003; 44:29-32.

3. Bebek N, Gürses C, Gokyigit A, Baykan B, Ozkara C, Dervent A. Hot water epilepsy: clinical and electrophysiologic findings based on 21 cases. Epilepsia 2001; 42:1180-1184.

4. Karan KR, Satishchandra P, Sinha S, Anand A. Rare SLC1A1 variants in hot water epilepsy. Hum Genet 2017; 136:693-703.

5. Yalçin AD, Toydemir HE, Forta H. Hot water epilepsy: clinical and electroencephalographic features of 25 cases. Epilepsy Behav 2006; 9:89-94.

6. Herzog AG, Drislane FW, Schomer DL, Pennell PB, Bromfield EB, Dworetzky BA, et al. Differential effects of antiepileptic drugs on sexual function and hormones in men with epilepsy. Neurology 2005; 65:1016-1020.

7. Atarodi-Kashani Z, Kariman N, Ebadi A, Alavi Majd H, BeladiMoghadam N. Sexual function and related factors in Iranian woman with epilepsy. Seizure 2017; 52:147-153.
8. Yogarajah M, Mula M. Sexual Dysfunction in Epilepsy and the Role of Anti-Epileptic Drugs. Curr Pharm Des 2017; 23:56495661.

9. Rathore C, Henning OJ, Luef G, Radhakrishnan K. Sexual dysfunction in people with epilepsy. Epilepsy Behav 2019; 100:106495.

10. Onat F, Eskazan E. Antiepileptic drugs: In Bora I, Gurses C, Yeni N (editors). Epilepsi. First ed., Nobel Tip Kitabevleri, 2008, 595607. (Turkish)

11. Reis RM, de Angelo AG, Sakamoto AC, Ferriani RA, Lara LA. Altered sexual and reproductive functions in epileptic men taking carbamazepine. J Sex Med 2013; 10:493-499.

12. Wu D, Chen L, Ji F, Si Y, Sun H. The effects of oxcarbazepine, levetiracetam, and lamotrigine on semen quality, sexual function, and sex hormones in male adults with epilepsy. Epilepsia 2018; 59:1344-1350.

13. Luef G, Krämer G, Stefan H. Oxcarbazepine treatment in male epilepsy patients improves pre-existing sexual dysfunction. Acta Neurol Scand 2009; 119:94-99.

14. Yang Y, Wang X. Sexual dysfunction related to antiepileptic drugs in patients with epilepsy. Expert Opin Drug Saf 2016; 15:31-42. 